\title{
Atomically Thin Molybdenum Disulfide Nanopores with High Sensitivity for DNA Translocation
}

\author{
Ke Liu, ${ }^{\dagger}$ Jiandong Feng, ${ }^{\dagger}$ Andras Kis, ${ }^{\dagger}$ and Aleksandra Radenovic ${ }^{\dagger, *}$ \\ ${ }^{\dagger}$ Laboratory of Nanoscale Biology, Institute of Bioengineering, School of Engineering, EPFL, 1015 Lausanne, Switzerland, and ${ }^{\ddagger}$ Laboratory of Nanoscale Electronics \\ and Structure, Institute of Electrical Engineering, School of Engineering, EPFL, 1015 Lausanne, Switzerland
}

\section{ABSTRACT}

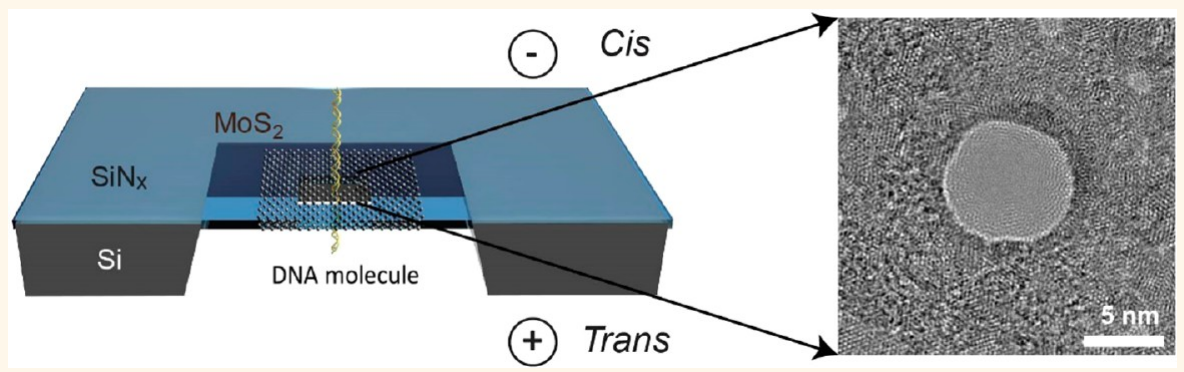

Atomically thin nanopore membranes are considered to be a promising approach to achieve single base resolution with the ultimate aim of rapid and cheap DNA sequencing. Molybdenum disulfide $\left(\mathrm{MoS}_{2}\right)$ is newly emerging as a material complementary to graphene due to its semiconductive nature and other interesting physical properties that can enable a wide range of potential sensing and nanoelectronics applications. Here, we demonstrate that monolayer or

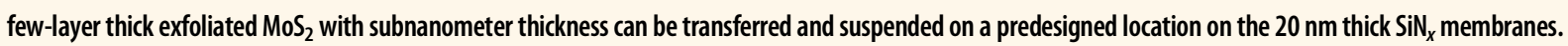
Nanopores in $\mathrm{MoS}_{2}$ are further sculpted with variable sizes using a transmission electron microscope (TEM) to drill through suspended portions of the MoS membrane. Various types of double-stranded (ds) DNA with different lengths and conformations are translocated through such a novel architecture, showing improved sensitivity (signal-to-noise ratio $>10$ ) compared to the conventional silicon nitride $\left(\mathrm{SiN}_{x}\right)$ nanopores with tens of nanometers thickness. Unlike graphene nanopores, no special surface treatment is needed to avoid hydrophobic interaction between DNA and the surface. Our results imply that MoS 2 membranes with nanopore can complement graphene nanopore membranes and offer potentially better performance in transverse detection.

KEYWORDS: nanopores $\cdot 2 \mathrm{D}$ materials $\cdot$ dichalcogenides $\cdot \mathrm{MoS}_{2} \cdot \mathrm{DNA} \cdot$ single molecules

S olid-state nanopores exhibit relatively lower single molecule detection sensitivity compared to biopores due to their intrinsic thickness and lack of control over surface charge distribution. During a typical translocation experiment in $30 \mathrm{~nm}$ thick $\mathrm{SiN}_{x}$ membranes, DNA regions approximately $30 \mathrm{~nm}$ long and containing $\sim 100$ base pairs (bps) are residing within a nanopore at any given time. Therefore, single base resolution is not expected here. Recently, thin membranes have been proposed to extend the applications of solidstate nanopore to, e.g., detection of short DNA oligomers and differentiation of short nucleotide homopolymers. ${ }^{1,2}$ The merits of this novel approach are twofold. First, the thin membrane can amplify both baseline and signal amplitude without increasing noise levels, resulting in a greatly enhanced signal-to-noise ratio (SNR). Furthermore, ultrasmall nanopores ( 1 to $2 \mathrm{~nm}$ ) can be further adapted to mimic biological nanopores, where a narrow constriction (1.2 $\mathrm{nm}$ for MspA and $1.5 \mathrm{~nm}$ for $\alpha$-hemolysin) and small sensing length $(0.5 \mathrm{~nm})$ could facilitate single nucleotide identification along DNA strands. This concept can be exploited using the 2D material graphene, a single atomic layer of carbon $^{3}$ which can extend over macroscopic scales in two dimensions ( $\mathrm{mm}$ in size) while being atomically thin (few $\AA$ ) in the perpendicular dimension. Several groups already demonstrated the use of graphene as a nanopore membrane for detecting DNA translocation. ${ }^{4-7}$ With the
* Address correspondence to aleksandra.radenovic@epfl.ch.

Received for review November 26, 2013 and accepted February 4, 2014.

Published online

10.1021/nn406102h

(c) XXXX American Chemical Society 
use of a modern transmission electron microscope (TEM), nanopores in graphene can be sculpted atom by atom with diameters that can be tailored for various applications. ${ }^{7-9}$ Another advantage for graphene, as predicted by theoretical calculations, ${ }^{10-12}$ is its potential use in a transistor integrated with a nanopore where DNA translocation can modulate the tunneling current or gate the transistor channel. We recently demonstrated the first realization of simultaneous detection of DNA translocation with two synchronized signals, the ionic current in the nanopore and local potential change in the graphene nanoribbon transistor. ${ }^{13}$ Apart from all these encouraging achievements, it is worth noting that the strong $\pi-\pi$ interaction between graphene and DNA ${ }^{14}$ leads to undesirable adsorption of DNA on graphene, which may hinder the DNA translocation through graphene nanopores. Some groups have exploited surface modification, ${ }^{15}$ atomic layer deposition ${ }^{5}$ and high $\mathrm{pH}$ and ionic strengths ${ }^{4}$ to minimize surface interaction. The first two approaches ultimately increase the sensing length to few nanometers which is not desirable for single nucleotide resolution. An alternative solution is to use other $2 \mathrm{D}$ materials such as insulating boron nitride $(\mathrm{BN})^{16}$ as the membrane material. To the best of our knowledge, this letter is the first example of utilizing $\mathrm{MoS}_{2}$, a newly emerging transition metal dichalcogenide-based material, as a nanopore membrane.

$\mathrm{MoS}_{2}$ has drawn attention as a promising material with potential applications complementary to graphene due to the presence of a bandgap and versatile chemistry, ${ }^{17}$ which makes it attractive in various applications including catalysis, energy storage, sensing and electronic devices such as field-effect transistors ${ }^{18}$ and logic circuits. ${ }^{19}$ The thickness of single-layer $\mathrm{MoS}_{2}$ is $\sim 6.5 \AA$, comparable to the thickness of graphene (3.5 $\AA$ ) and the spacing between two neighboring nucleotides along ssDNA (3.2-5.2 ̊). Both exfoliation ${ }^{20}$ and chemical vapor deposition (CVD) ${ }^{21,22}$ can be used to produce thin layers of $\mathrm{MoS}_{2}$ with good quality. ${ }^{20-22}$ In this letter, we propose that $\mathrm{MoS}_{2}$ can be used as an inorganic analogue of graphene membrane for nanopore-based DNA biosensing. To make nanopores in free-standing $\mathrm{MoS}_{2}$ membranes, we use a sophisticated transfer method ${ }^{23}$ to suspend monolayer and few-layer $\mathrm{MoS}_{2}$ on $\mathrm{SiN}_{x}$ supporting membranes and exploit the state-of-art high resolution electron microscopy technique to sculpt nanopores in variable diameters. Such membranes can be used to detect DNA translocation with high SNR (>10) and 5-fold enhancement in the ionic current signal. Realization of DNA translocations through an $\mathrm{MoS}_{2}$ nanopore membrane imply that it can compete with graphene nanopore membrane in terms of spatial resolution and potentially better performance when acting as a transistor integrated with a nanopore, allowing transverse detection of DNA translocations. ${ }^{13}$

\section{RESULTS AND DISCUSSION}

The experimental concept is schematized in Figure 1a, where few layers or even monolayer $\mathrm{MoS}_{2}$ are suspended on the pre-etched square-shaped opening on $20 \mathrm{~nm}$ thick supporting $\mathrm{SiN}_{x}$ membranes. Thus, DNA can translocate through subnanometer thick $\mathrm{MoS}_{2}$ instead of $20 \mathrm{~nm}$ thick $\mathrm{SiN}_{x}$ to achieve a better spatial resolution. We used the widely adopted micromechanical exfoliation method ${ }^{3}$ to exfoliate few-layer $\mathrm{MoS}_{2}$ from natural $\mathrm{MoS}_{2}$ bulky material onto the surface of substrates covered with $270 \mathrm{~nm} \mathrm{SiO}_{2}$ chips with fiducial markers. An optical microscope is used to identify single and few-layer $\mathrm{MoS}_{2}$ by their contrast under illumination. ${ }^{24}$ As shown in Figure $1 \mathrm{~b}$, monolayer $\mathrm{MoS}_{2}$ shows minimum contrast with respect to the substrate. The coordinates of chosen flakes were recorded and used for the further transfer onto the $\operatorname{SiN}_{x}$ membrane. To verify the thickness of this chosen flake, we used atomic force microscopy (AFM) to obtain its height profile, as shown in Figure 1d. The thickness is $9 \AA$ from AFM measurements and is indicative of a monolayer, which is consistent with the optical observation. Subsequently, this flake was transferred from the silicon dioxide substrate to a square-shaped opening (ranging from 200 to $500 \mathrm{~nm}$ in size to reduce electric noise when the flake comes in contact with ionic buffer, ${ }^{4}$ see Figure S1a) on the target $\mathrm{SiN}_{x}$ supporting membrane using a standard graphene transfer method. ${ }^{23}$ Figure $1 \mathrm{c}$ is the optical image after a successful transfer of the flake shown in Figure $1 b, d$ to the desired location (marked by the black circle) on the supporting membrane. We navigated in the TEM with low magnification to search for the chosen flake. Figure 1e illustrates the full coverage of the opening in $\mathrm{SiN}_{x}$ by the flake (marked by the black circle), preventing ionic current leakage. Figure S1b demonstrates an example of unsuccessful transfer, where the $\mathrm{MoS}_{2}$ flake is not covering the square-shaped opening. The $\mathrm{MoS}_{2}$ lattice can be clearly resolved in the highmagnification image, shown in Figure S2a, with the diffraction pattern (DP) reflecting the hexagonal symmetry of $\mathrm{MoS}_{2}{ }^{25}$ as shown in the inset of Figure S2a. The drilling process lasts only for several seconds after which a nanometer-sized pore appears. This is another indication that we only drill through few atoms of $\mathrm{MoS}_{2}$. Therefore, a good thermal and mechanical stability is highly preferred for such a short drilling period, especially in the case of small pores. We prefer to blank the beam for several minutes before the drilling process in order to minimize the drift for the both beam and the sample. Figure $1 f-i$ shows several examples of nanopores with various sizes. Therefore, we want to infer that nanopores can be sculpted with the flexibility for pore dimensions using a highly focused electron beam. Moreover, as shown in Figure S2b, in some cases the number of layers can be identified by 
a
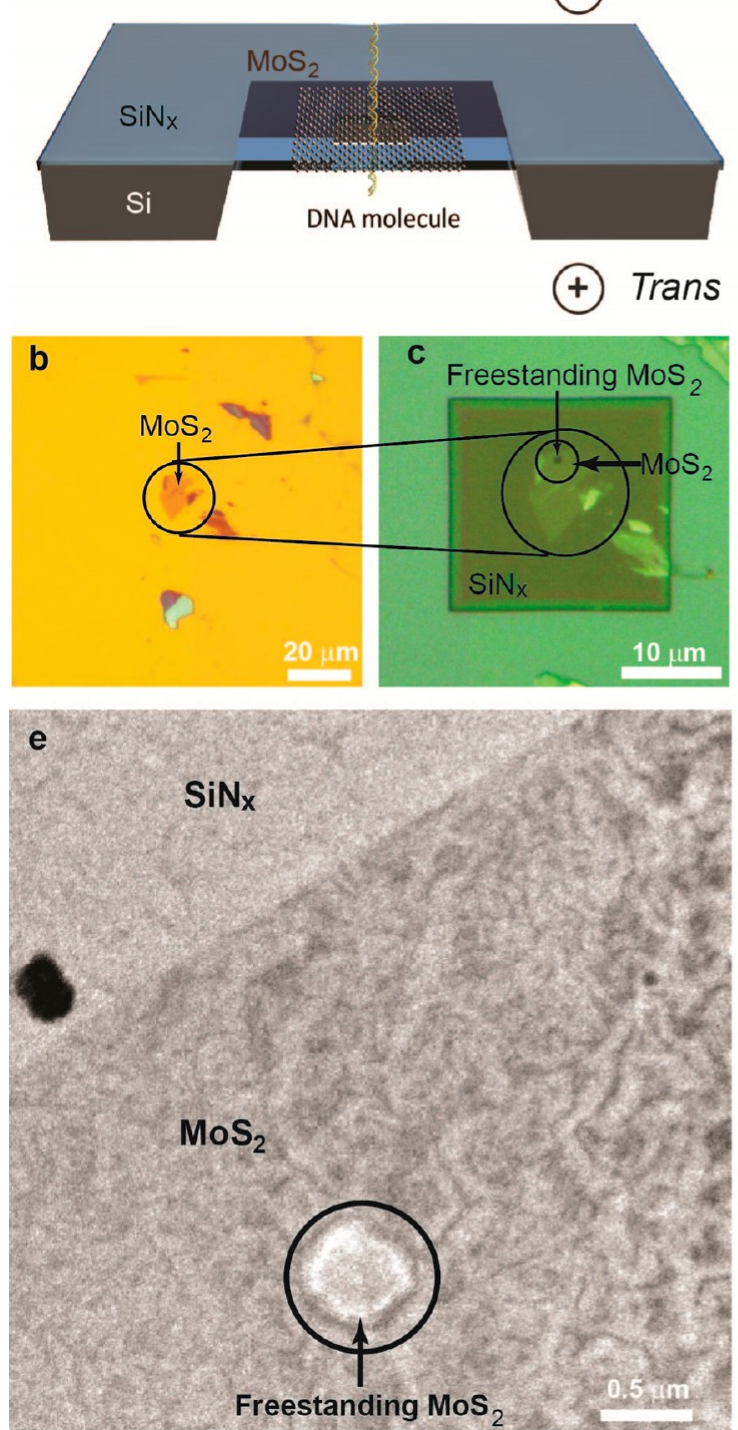

d
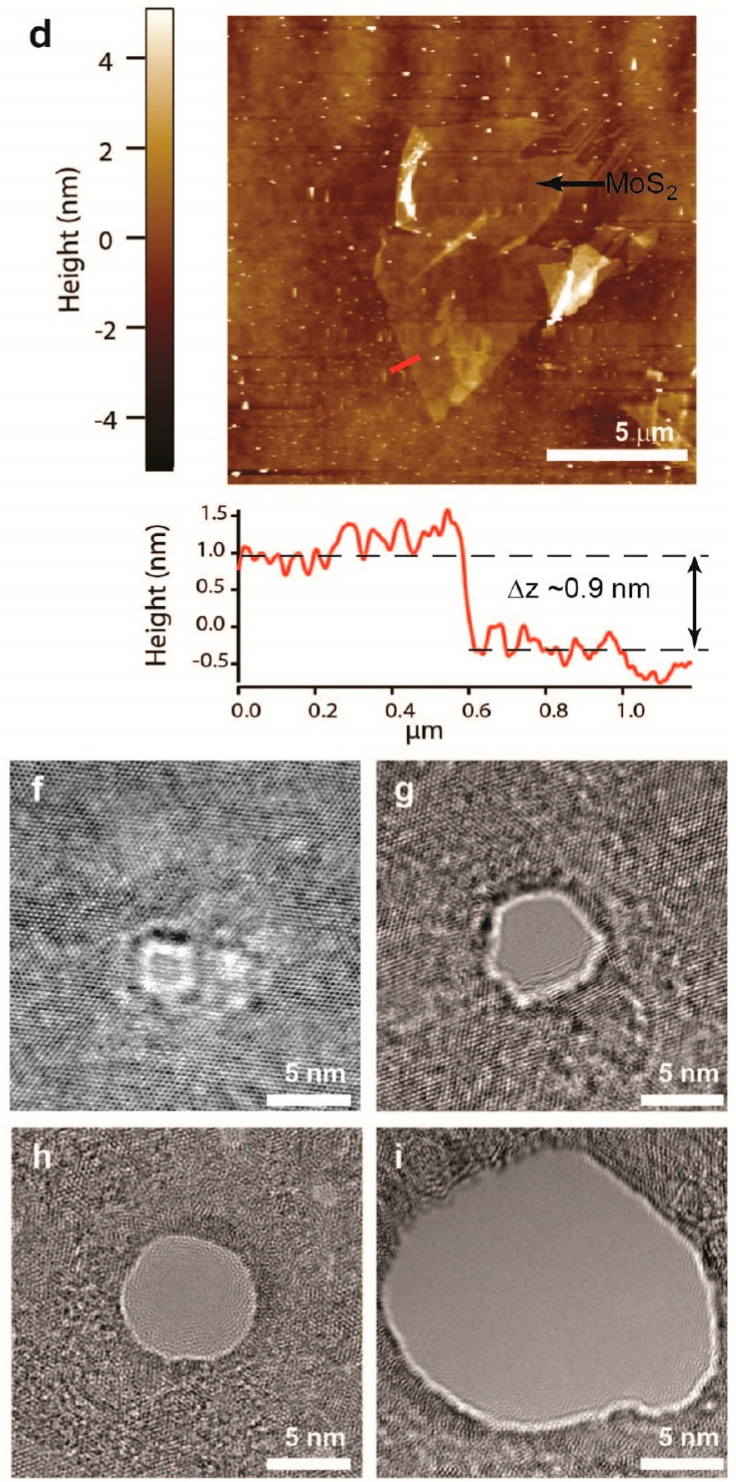

Figure 1. (a) Schematic illustration of an $\mathrm{MoS}_{2}$ nanopore membrane for DNA translocation. Monolayer $\mathrm{MoS}_{2}$ is suspended on a $\mathrm{SiN}_{x}$ supporting membrane that separates two reservoirs containing buffered potassium chloride. Electrical field is applied by a pair of $\mathrm{Ag} / \mathrm{AgCl}$ electrodes to drive DNA molecules passing through a nanometer size pore on $\mathrm{MoS}_{2}$, while ionic current through the pore is recorded using an Axonpatch low-noise amplifier. (b) Optical image of a freshly exfoliated monolayer $\mathrm{MoS}_{2}$ flake (marked with the black circle) exhibiting minimum contrast with respect to the $\mathrm{SiO}_{2}$ substrate. (c) Optical image after the chosen flake has been transferred from the $\mathrm{SiO}_{2}$ substrate to the desired location (a square-shaped opening made by $\mathrm{EBL}$ and RIE) on the $\mathrm{SiN}_{x}$ supporting membrane. (For fabrication details see Methods section and Supporting Information.) (d) AFM image of the chosen flake in (b). Height profile is taken from the red line in the image, showing a $9 \AA$ A height difference between the surface and the $M_{0} S_{2}$ flake. (e) Low-magnification TEM image of a $M_{0} S_{2}$ flake fully covering the opening in the $\mathrm{SiN}_{x}$ membrane marked with the black circle. The edge of this flake is clearly seen in the upper part of the image. (f-i) Highresolution TEM images of nanopores with various sizes drilled by a focused electron beam. The lattice of $\mathrm{MoS}_{2}$ is visible under such a magnification (1 MX).

inspecting the folded edges of the flake. Nevertheless, we routinely used optical microscopy to choose thin flakes because it is straightforward and less time-consuming.

We start by investigating the current-voltage (IV) characteristics of $\mathrm{MoS}_{2}$ nanopores with various sizes $(2-20 \mathrm{~nm})$ immersed in the $2 \mathrm{M} \mathrm{KCl}$ buffered solution. Figure 2a,b shows examples of IV curves measured in the $\mathrm{KCl}$ buffer, exhibiting linear and symmetric characteristics from -500 to $500 \mathrm{mV}$. According to the model first proposed by Wanunu et al. ${ }^{1}$ and later adopted by Kowalcyzk et al., ${ }^{26}$ the conductance of nanopore can be described by eq 1 ,

$$
G=\sigma\left[\frac{4 L}{\pi d^{2}}+\frac{1}{d}\right]^{-1}
$$

where $\sigma, L$ and $d$ are the ionic conductivity of $2 \mathrm{M} \mathrm{KCl}$ $\left(20 \mathrm{~S} \mathrm{~m}^{-1}\right)$, membrane thickness and nanopore diameter, respectively. Two major elements associated with pore geometry contribute to the conductance, namely channel resistance (the first term in the 

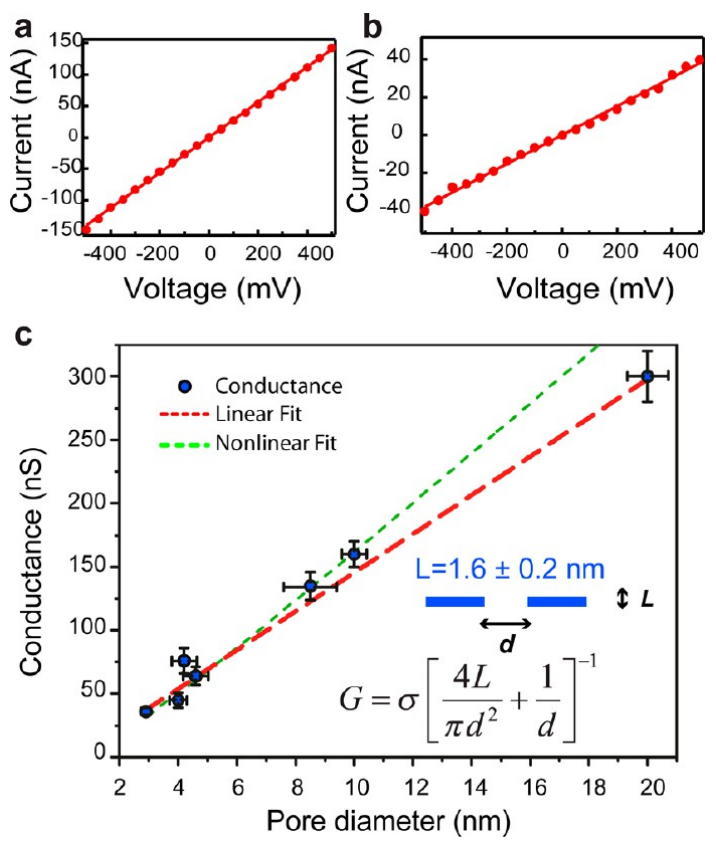

Figure 2. (a) Current-voltage characteristic of a $20 \mathrm{~nm} \mathrm{MoS}$ nanopore measured in $2 \mathrm{M} \mathrm{KCl}$. (b) Current-voltage characteristics of a $5 \mathrm{~nm} \mathrm{MoS}$ nanopore measured in $2 \mathrm{M} \mathrm{KCl}$. (c) Correlation between pore diameters and their conductances. Conductances are derived from linear fits of IV measurements in $2 \mathrm{M} \mathrm{KCl}$ with bias voltage swept from -0.5 to $+0.5 \mathrm{~V}$. To exclude either leaking pores $G>300 \mathrm{nS}$ or clogged pores $G<10 \mathrm{nS}$, we used only devices displaying conductances higher than $10 \mathrm{nS}$ and lower than $300 \mathrm{nS}$. Error bars of the pore diameters indicate the asymmetry of the pores. Inset shows a simple scheme for the thin membrane with a nanopore and related equation to describe conductance, where $G$ is conductance, $\sigma$ is ionic conductivity, $L$ is thickness and $d$ is pore diameter. The nonlinear fitting (green line) is based on the eq 1 shown in the inset to subtract $L$. And the linear fitting (red line) is based on the simplified eq 2, $\mathbf{G}=\sigma d$, to subtract $\sigma$.

equation) and access resistance (the second term in the equation). Figure $2 c$ shows a plot of all working devices in this study. Using a nonlinear fit, we find $L$ with a value of $1.6 \pm 0.2 \mathrm{~nm}$, reflecting an atomically thin feature of the $\mathrm{MoS}_{2}$ membranes. For an ultrathin membrane, channel resistance is much smaller than the access resistance. Therefore, the conductance can also be expressed as eq 2 :

$$
G=\sigma d
$$

As a result, a nearly linear relationship between pore conductance and pore diameter is expected. From the linear fit, we find $\sigma$ with a value of $17.5 \pm 1.5 \mathrm{~S} \mathrm{~m}^{-1}$, which is in a good agreement with ionic conductivity of $2 \mathrm{M} \mathrm{KCl}\left(20 \mathrm{~S} \mathrm{~m}^{-1}\right)$. Therefore, both fits are suitable. At any given pore size, the conductance from the $\mathrm{MoS}_{2}$ nanopore is much larger than that of $\mathrm{SiN}_{x}$. Moreover, we did not observe an influence of the number of layers (always less than 4) on the conductance since the pore diameter is larger than its thickness.

Ideally, $\left(\mathrm{K}^{+}, \mathrm{Cl}^{-}\right)$ions flow in two directions under the influence of the electrical field through a nanopore, resulting in a constant ionic current, namely, the baseline current. DNA translocation will give rise to temporary blockades in ionic pore current manifested by a decrease in ionic current on the time-scale of approximately $100 \mu \mathrm{s}$ to $10 \mathrm{~ms}$, as shown in Figure 3a. We first translocated pNEB plasmid DNA through a $20 \mathrm{~nm}$ diameter $\mathrm{MoS}_{2}$ nanopore to eliminate the multiple conformation issue. Two parameters, the amplitude of blockage and dwell time are used to quantify individual translocation events. In our group, we developed a so-called cumulative sums (CUSUM) algorithm to detect events automatically ${ }^{27}$ and extract abovementioned parameters for each event. Events are concatenated with short segments of the baseline signal preceding and following them. Due to the circular shape of the PNEB plasmid, all events have only one level indicating a single conformation. The signal amplitude also increases upon raising the applied voltage as shown in Figure $3 \mathrm{~b}$. Mean signal amplitudes are 0.3, 0.7, 0.9, and $1.1 \mathrm{nA}$ for 100, 200, 300 , and $400 \mathrm{mV}$, respectively. Here, we used $2^{*}(2.2 / d)^{2}$ to calculate theoretical blockage, where $d=20 \mathrm{~nm}$ in this case. Blockage percentage is $2.4 \%$, in accordance with our experimental value of $1.5 \%$. Scatter plots are used to describe the statistics of DNA translocation as shown in Figure 3c. For voltages above $200 \mathrm{mV}$, fast translocation is observed with a most probable dwell time of $\sim 100 \mu \mathrm{s}^{28}$ But for $100 \mathrm{mV}$, a much broader distribution (200 $\mu \mathrm{s}$ full-width at half-maximum) is observed with a mean dwell time of $\sim 300 \mu \mathrm{s}$, which is consistent with published results with graphene nanopores using DNA molecules of similar length. ${ }^{4-7}$ More insight can be gained by using a constant electron charge deficit (ECD) method $^{29}$ to fit the scatter plot (Figure 3c) for various voltages. As a result, a value of $\sim 500$ ke is obtained, which is at least five times of previously reported data for $3 \mathrm{kbp}$ DNA. ${ }^{30}$ This increase is mainly due to the increased blockage amplitude because of the greatly improved sensitivity of $\mathrm{MoS}_{2}$ nanopores compared to conventional $\mathrm{SiN}_{x}$ nanopores. In ref 30, for the circular plasmid DNA, the current drop is $\sim 100 \mathrm{pA}$ at $100 \mathrm{mV}$, whereas we got $\sim 400 \mathrm{pA}$ at $100 \mathrm{mV}$. Garaj et al. also reported from 2-fold up to 10-fold enhancement in signal for DNA translocation in graphene nanopores. ${ }^{4,7}$ Therefore, we conclude that $\mathrm{MoS}_{2}$ and graphene nanopores have comparable sensitivities. It is very important to have a good signal-to-noise ratio (SNR), preferably more than 6 , for event detection. Here, we obtain SNR > 10 (100 pA RMS noise and $\sim$ nA signal). The percentage of device failure (conductance higher than 300 $\mathrm{nS}$ due to leakage or lower than $10 \mathrm{nS}$ due to pore clogging) in solution is surprisingly low $(<30 \%)$. A very important feature of $\mathrm{MoS}_{2}$ membranes when compared to graphene ones is that undesirable adsorption of DNA onto surface is eliminated here, while many additional surface treatments were needed in order to reduce the strong hydrophobic interaction between DNA and graphene. ${ }^{4-7}$ 

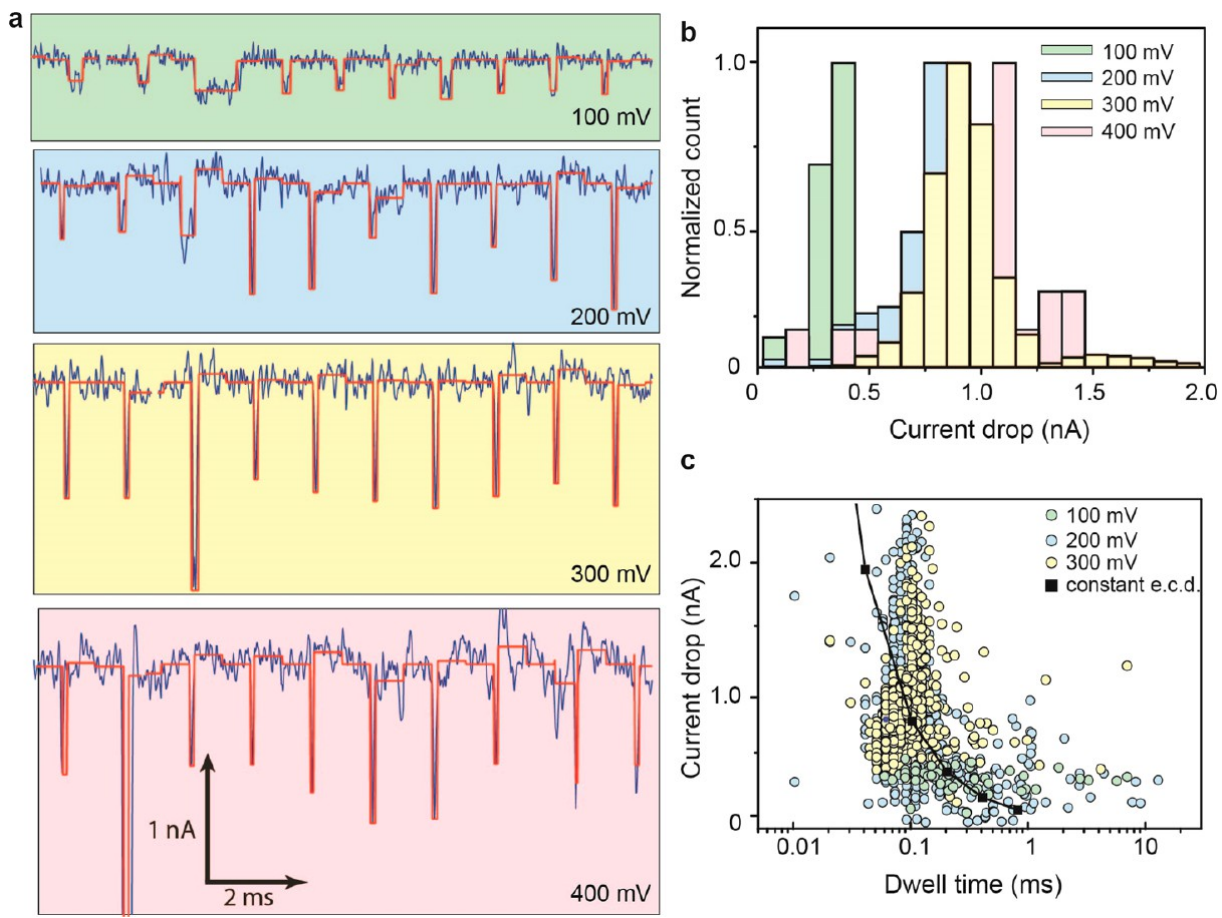

Figure 3. (a) Concatenated events of pNEB plasmid DNA translocating a $20 \mathrm{~nm} \mathrm{MoS}$ nanopore in $2 \mathrm{M} \mathrm{KCl}$. Raw signal is in blue and fits are shown in red. Fits are performed using a custom "OpenNanopore" Matlab code. ${ }^{27}$ (b) Normalized distribution of current amplitudes at various voltages. (c) Scatter plots of 59, 1823, and 1642 events for 100, 200, and $300 \mathrm{mV}$, respectively. Events at $400 \mathrm{mV}$ are shown in the Supporting Information in comparison with a $5 \mathrm{~nm}$ nanopore in Supporting Information Figure S3. Each event is represented by its dwell time and current drop. An electron charge deficit (ECD) method is used to fit the area of individual events.

To gain more understanding on the interaction between $\mathrm{MoS}_{2}$ and DNA, we translocated pNEB DNA through a $5 \mathrm{~nm}$ diameter $\mathrm{MoS}_{2}$ pore. Representative concatenated traces are shown in Figure S3a where the same experimental conditions were used except pore diameter which was $5 \mathrm{~nm}$. From the scatter plot in Figure S3b, both mean current amplitude and mean dwell time are larger for the $5 \mathrm{~nm}$ pore compared to the $20 \mathrm{~nm}$ pore, implying a local interaction between the edge of the $\mathrm{MoS}_{2}$ pore and the DNA molecule. To extend this statement, this interaction happens only when DNA is sliding through the edge of pore with the effect of retarding DNA translocation. For larger pores $(20 \mathrm{~nm})$, translocations tend to occur in a frictionless manner.

As first seen using solid-state nanopores, DNA conformations can be revealed using the quantization of current blockage when DNA translocates through a nanopore. ${ }^{31}$ Here, we used $\lambda$-DNA, which has a wealth of secondary structures and conformations, to test the performance of $\mathrm{MoS}_{2}$ pores in distinguishing between them by ionic current measurements. Figure 4 a shows two typical current vs time traces for DNA translocation through $\mathrm{SiN}_{x}$ and $\mathrm{MoS}_{2}$ pores with similar sizes of $20 \mathrm{~nm}$, where much bigger current dips are clearly visible for $\mathrm{MoS}_{2}$ pores showing greater SNR. To facilitate comparison in conductance drops, we have chosen relatively large diameter pores $20 \mathrm{~nm}$ in order to minimize DNA-nanopore wall interactions that could be different for $\mathrm{MoS}_{2}$ nanopores $0.7 \mathrm{~nm}$ thin compared to $20 \mathrm{~nm}$ thick SiNx nanopores After careful inspection of the events, we selected four events to illustrate major conformations occurring during $\lambda$-DNA translocation. Specifically, in Figure $4 b$, the event 1 has a conductance drop of $\sim 5 \mathrm{nS}$, which is attributed to the translocation in an unfolded or linear manner. It is worth noting that a 5 -fold enhancement in signal amplitude is observed compared to the typical conductance drop of $\sim 1 \mathrm{nS}$ in $\mathrm{SiN}_{x}$ platforms. For the event 2 , DNA enters the pore in a folded manner manifested by a conductance drop of $\sim 10 \mathrm{nS}$ and then translocates in an unfolded manner with a conductance drop of $\sim 5 \mathrm{nS}$. For the event 3 , DNA is in a folded configuration during the whole translocation manifested by a conductance drop of $\sim 10 \mathrm{nS}$. Sometimes, we saw very deep current dips in the very beginning of an event, indicating a "bumping" of DNA onto the orifice of the pore in a coiled form (event 4 in Figure 4b). After entering the pore, the DNA molecule is stretched under electric field and it results in a conductance drop of $\sim 5 \mathrm{nS}$. The statistics of these events are presented using a scatter plot in Figure 4c. Moreover, a mean dwell time of $\sim 1 \mathrm{~ms}$ can be obtained, which is larger than that of shorter pNEB and is expected for longer $\lambda$-DNA.

The stability and durability of $\mathrm{MoS}_{2}$ nanopore membranes are also tested. To maintain a good SNR in this study, we apply voltages in the range from 200 to $400 \mathrm{mV}$. Although we observe translocation data at 


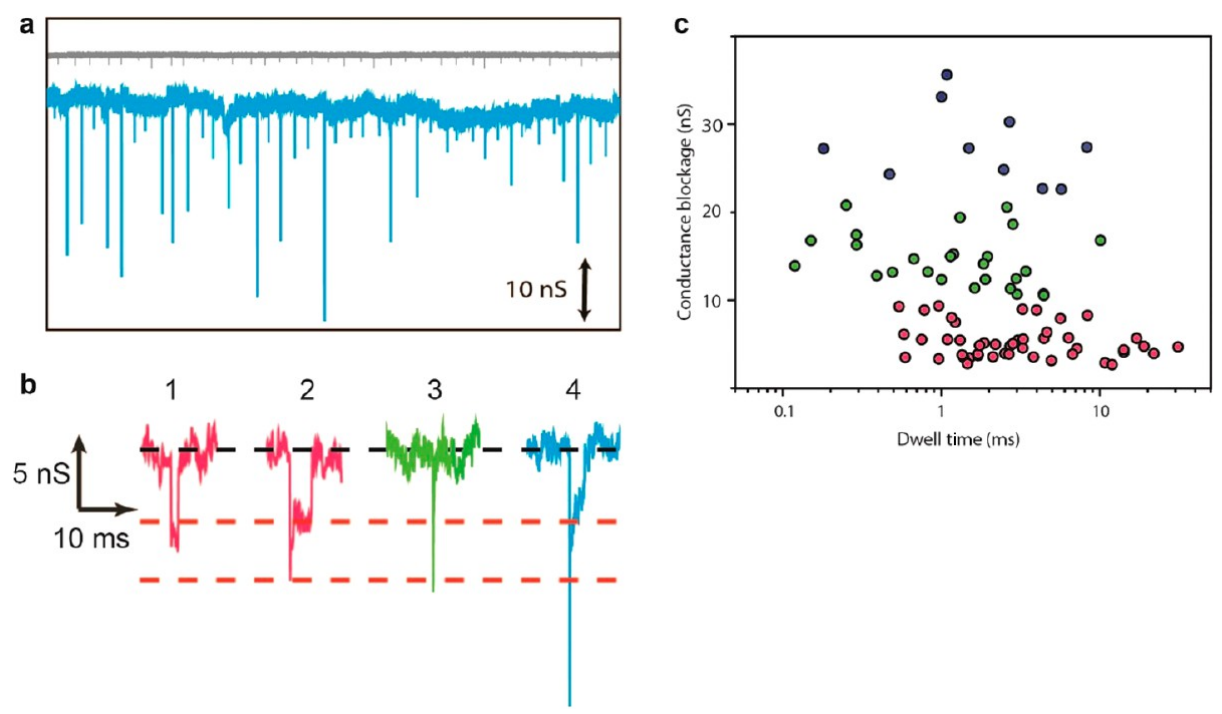

Figure 4. (a) A representative trace (in blue) of $\lambda$-DNA translocation through a $20 \mathrm{~nm} \mathrm{MoS}$ nanopore in $2 \mathrm{M} \mathrm{KCl}$ under a $200 \mathrm{mV}$ bias voltage. The upper trace (in black) is an example of $\lambda$-DNA translocation through a SiN $_{x}$ nanopore. (b) Selected individual events with quantized current drops implying multiple conformations of $\lambda$-DNA within the nanopore, i.e., unfolded (1), partially folded (2), folded (3), and bumping event (4). (c) Scatter plot of 104 events. Each event is represented by its dwell time and current blockage. Events statistics are collected from two devices.

$800 \mathrm{mV}$ (data not shown here), we observed that ionic current in some devices starts to be unstable, therefore in practice we avoided application of voltages higher than $400 \mathrm{mV}$. Compared to nanopores of other 2D materials, ${ }^{7,15,16} \mathrm{MoS}_{2}$ has a wider window of the applied voltage in the ionic buffer. Our devices can work for hours without permanent clogging. ${ }^{15}$ As shown in Figure S4, the device can operate for $9 \mathrm{~h}$ without degradation or saturation in the conductance. Translocations can be still observed after $9 \mathrm{~h}$ working in the ionic buffer. Generally, thousands of events can be collected in a single device, depending on the concentration of the analyte and the applied bias.

As for graphene nanopores, no significant slowing down can be achieved even with small-diameter pores $(\sim 2 \mathrm{~nm})$. The velocity of DNA translocation is $\sim 20 \mathrm{~ns} / \mathrm{bp}$, still beyond the present amplification bandwidth of 1 $\mathrm{MHz}$ (Chimera Instruments, New York, NY, USA) for translocation experiments. Although the enhancement of signal amplitude is dramatic in $\mathrm{MoS}_{2}$ pore, the lack of temporal resolution is the major obstacle that should be overcome for wider applications. An important advantage of $\mathrm{MoS}_{2}$ over graphene and boron nitride is that the intrinsic bandgap nature of $\mathrm{MoS}_{2}$ renders the implementation of sequencespecific transistors more promising. The noise in $\mathrm{MoS}_{2}$ nanopores is higher than that of $\operatorname{SiN}_{x}$ as shown in Figure $4 a$. Such an enhancement of the noise was also reported for graphene nanopores. ${ }^{4-7}$ To suppress noise and further improve SNR, we suggest curing polydimethylsiloxane (PDMS) on the nanopore support chip while leaving the $\mathrm{MoS}_{2}$ nanopore exposed. $^{32}$

\section{CONCLUSION}

To conclude, we present a versatile method of producing nanopore membranes based on $\mathrm{MoS}_{2}$. The starting material $\mathrm{MoS}_{2}$ is carefully characterized by optical imaging, AFM and TEM. As a result, membranes with a single size-tunable nanopore can be produced with good yield and very low device failure rate when working in high ionic strength buffers. Translocation of various types of DNA exhibits a signal amplitude that is five times higher than in the case of solid-state $\mathrm{Si}_{3} \mathrm{~N}_{4}$ membranes and a SNR of more than 10. These features are highly desirable for event detection and we take advantage of them by showing the electric-field induced unfolding of a $48 \mathrm{kbp}$ long DNA molecule within the nanopore which manifests itself in the quantization of the current drop. Unlike graphene nanopores, no special surface treatment is needed to avoid strong interaction between DNA and the surface. Our results imply that $\mathrm{MoS}_{2}$ nanopore membranes can compete with graphene nanopore membranes in terms of spatial resolution and possibly better performance for transverse detection. ${ }^{13}$

\section{METHODS}

The $20 \mathrm{~nm}$ thick supporting $\mathrm{SiN}_{x}$ membranes are manufactured in a standard procedure ${ }^{33}$ using anisotropic $\mathrm{KOH}$ etching. Membrane sizes vary from 10 to $50 \mu \mathrm{m}$ depending on the size of the backside opening. Electron beam lithography (EBL) and reactive ion etching (RIE) is used to make a square-shaped opening with a size of 200-500 nm on the membrane. $\mathrm{MoS}_{2}$ flakes are first mechanically exfoliated onto substrates with 
$270 \mathrm{~nm} \mathrm{SiO} 2$ and fiducial markers. Next we use optical microscope (Olympus IX51) to identify few layers or even monolayer flakes by their optical contrast. ${ }^{24}$ The thickness of chosen flakes is further confirmed by AFM measurements (Asylum Research Cypher). The method of transferring flakes to the square-shaped opening located on the $\mathrm{SiN}_{x}$ membrane is similar to the widely used graphene transfer method. ${ }^{23}$ Electron beam drilling ${ }^{34-36}$ is performed in a JEOL 2200FS TEM operated at an acceleration voltage of $200 \mathrm{kV}$. Before loading in the microscope, the samples are annealed at $400{ }^{\circ} \mathrm{C}$ under a $\mathrm{H}_{2} / \mathrm{Ar}$ flux in order to remove any residual organic material left on the surface from the microfabrication processing and prevent hydrocarbon deposition. ${ }^{37}$ The fabrication process is detailed as a process flow shown in the Supporting Information. Membranes are imaged in the TEM mode with low magnification $(<10 \mathrm{k} \times)$ in order to identify the location of suspended $\mathrm{MoS}_{2}$ flake. Drilling is performed by focusing the beam with the condensor lens aperture $(\mathrm{CLA})$ at high magnification $(600 \mathrm{k} \times$ to $1 \mathrm{M} \times)$. The nanopore membrane chip is mounted inside custom flow cell as soon as possible after drilling, otherwise stored in a desiccator with controlled humidity. After mounting the sample in the microfluidic setup, the wetting of the pore is facilitated by flushing the microfluidic system with a water-ethanol (v/v, 1:1) solution. It is crucial to inspect and remove bubbles trapped in the microfluidic channels. An Axopatch 200B patch clamp amplifier (Molecular Devices, Inc., Sunnyvale, CA) is used to record the ionic current in the single cell configuration with a sampling rate of $100 \mathrm{kHz}$ and lowpass filter of $10 \mathrm{kHz}$. We use a NI PXI-4461 card for data digitalization and custom-made LabView software for data acquisition. Chlorinated $\mathrm{Ag} / \mathrm{AgCl}$ electrodes are inserted in both cis and trans reservoirs and connected to the Axopatch 200B. DNA samples (pNEB193, plasmid $2.7 \mathrm{kbp}$, New England; $\lambda$-DNA, 48 kbp, New England) are buffered with filtered and degassed $2 \mathrm{M} \mathrm{KCl}, 10 \mathrm{mM}$ Tris, $1 \mathrm{mM}$ EDTA and $\mathrm{pH} 7.4$ and adjusted to a final concentration of $1-10 \mathrm{ng} / \mu \mathrm{L}$. Finally, the solution containing DNA is injected into the cis chamber of the flow cell, which is grounded using the $\mathrm{Ag} / \mathrm{AgCl}$ electrode. Each type of DNA is translocated in at least two different devices and representative and reproducible results and analysis are presented. Data analysis is performed offline using a custom open source Matlab code, named OpenNanopore (http://lben.epfl.ch/page-79460-en.html), for event detection. $^{27}$

Conflict of Interest: The authors declare no competing financial interest.

Acknowledgment. This work was financially supported by European Research Council (grant no. 259398, PorABEL: Nanopore integrated nanoelectrodes for biomolecular manipulation and sensing). We thank the Centre Interdisciplinaire de Microscopie Electronique (CIME) at EPFL for access to electron microscopes; special thanks to D. T .L. Alexander for providing training and technical assistance with TEM. Devices fabrication was partially carried out at the EPFL Center for Micro/Nanotechnology (CMi). K.L. thanks Mr. Oriol Lopez Sanchez for the training in transfer techniques. We thank all the members from LBEN and LANES for assistance and discussion.

Supporting Information Available: Process flow; additional TEM observations; pNEB translocation dependence on the pore size; pore conductance as a function of time. This material is available free of charge via the Internet at http://pubs.acs.org.

\section{REFERENCES AND NOTES}

1. Wanunu, M.; Dadosh, T.; Ray, V.; Jin, J. M.; McReynolds, L.; Drndic, M. Rapid Electronic Detection of Probe-Specific Micrornas Using Thin Nanopore Sensors. Nat. Nanotechnol. 2010, 5, 807-814.

2. Venta, K.; Shemer, G.; Puster, M.; Rodriguez-Manzo, J. A.; Balan, A.; Rosenstein, J. K.; Shepard, K.; Drndic, M. Differentiation of Short, Single-Stranded DNA Homopolymers in Solid-State Nanopores. ACS Nano 2013, 7, 4629-4636.

3. Novoselov, K. S.; Geim, A. K.; Morozov, S. V.; Jiang, D.; Zhang, Y.; Dubonos, S. V.; Grigorieva, I. V.; Firsov, A. A.
Electric Field Effect in Atomically Thin Carbon Films. Science 2004, 306, 666-669.

4. Garaj, S.; Hubbard, W.; Reina, A.; Kong, J.; Branton, D.; Golovchenko, J. A. Graphene as a Subnanometre TransElectrode Membrane. Nature 2010, 467, 190-193.

5. Merchant, C. A.; Healy, K.; Wanunu, M.; Ray, V.; Peterman, N.; Bartel, J.; Fischbein, M. D.; Venta, K.; Luo, Z. T.; Johnson, A. T. C.; Drndic, M. DNA Translocation through Graphene Nanopores. Nano Lett. 2010, 10, 2915-2921.

6. Schneider, G. F.; Kowalczyk, S. W.; Calado, V. E.; Pandraud, G.; Zandbergen, H. W.; Vandersypen, L. M. K.; Dekker, C. DNA Translocation through Graphene Nanopores. Nano Lett. 2010, 10, 3163-3167.

7. Garaj, S.; Liu, S.; Golovchenko, J. A.; Branton, D. MoleculeHugging Graphene Nanopores. Proc. Natl. Acad. Sci. U.S.A. 2013, 110, 12192-12196.

8. Liu, S.; Zhao, Q.; Xu, J.; Yan, K.; Peng, H. L.; Yang, F. H.; You, L. P.; Yu, D. P. Fast and Controllable Fabrication of Suspended Graphene Nanopore Devices. Nanotechnology 2012, 23, 085301

9. Xu, Q.; Wu, M. Y.; Schneider, G. F.; Houben, L.; Malladi, S. K.; Dekker, C.; Yucelen, E.; Dunin-Borkowski, R. E.; Zandbergen, H. W. Controllable Atomic Scale Patterning of Freestanding Monolayer Graphene at Elevated Temperature. ACS Nano 2013, 7, 1566-1572.

10. Lagerqvist, J.; Zwolak, M.; Di Ventra, M. Fast DNA Sequencing via Transverse Electronic Transport. Nano Lett. 2006, 6, 779-782.

11. Zwolak, M.; Di Ventra, M. Colloquium: Physical Approaches to DNA Sequencing and Detection. Rev. Mod. Phys. 2008, 80, 141-165.

12. Postma, H. W. C. Rapid Sequencing of Individual DNA Molecules in Graphene Nanogaps. Nano Lett. 2010, 10, 420-425.

13. Traversi, F.; Raillon, C.; Benameur, S. M.; Liu, K.; Khlybov, S.; Tosun, M.; Krasnozhon, D.; Kis, A.; Radenovic, A. Detecting the Translocation of DNA through a Nanopore Using Graphene Nanoribbons. Nat. Nanotechnol. 2013, 8, 939945.

14. Husale, S.; Sahoo, S.; Radenovic, A.; Traversi, F.; Annibale, P.; Kis, A. Ssdna Binding Reveals the Atomic Structure of Graphene. Langmuir 2010, 26, 18078-18082.

15. Schneider, G. F.; Xu, Q.; Hage, S.; Luik, S.; Spoor, J. N.; Malladi, S.; Zandbergen, H.; Dekker, C. Tailoring the Hydrophobicity of Graphene for Its Use as Nanopores for DNA Translocation. Nat. Commun. 2013, 4, 2619.

16. Liu, S.; Lu, B.; Zhao, Q.; Li, J.; Gao, T.; Chen, Y.; Zhang, Y.; Liu, Z.; Fan, Z.; Yang, F.; You, L.; Yu, D. Boron Nitride Nanopores: Highly Sensitive DNA Single-Molecule Detectors. Adv. Mater. 2013, 25, 4549-4554.

17. Wang, Q. H.; Kalantar-Zadeh, K.; Kis, A.; Coleman, J. N.; Strano, M. S. Electronics and Optoelectronics of TwoDimensional Transition Metal Dichalcogenides. Nat. Nanotechnol. 2012, 7, 699-712.

18. Radisavljevic, B.; Radenovic, A.; Brivio, J.; Giacometti, V.; Kis, A. Single-Layer $\mathrm{MoS}_{2}$ Transistors. Nat. Nanotechnol. 2011, 6, 147-150.

19. Radisavljevic, B.; Whitwick, M. B.; Kis, A. Integrated Circuits and Logic Operations Based on Single-Layer $\mathrm{MoS}_{2}$. ACS Nano 2011, 5, 9934-9938.

20. Novoselov, K. S.; Jiang, D.; Schedin, F.; Booth, T. J.; Khotkevich, V. V.; Morozov, S. V.; Geim, A. K. Two-Dimensional Atomic Crystals. Proc. Natl. Acad. Sci. U.S.A. 2005, 102, 1045110453.

21. Zhan, Y. J.; Liu, Z.; Najmaei, S.; Ajayan, P. M.; Lou, J. LargeArea Vapor-Phase Growth and Characterization of $\mathrm{MoS}_{2}$ Atomic Layers on a $\mathrm{SiO}_{2}$ Substrate. Small 2012, 8, 966-971.

22. Liu, K. K.; Zhang, W. J.; Lee, Y. H.; Lin, Y. C.; Chang, M. T.; Su, C.; Chang, C. S.; Li, H.; Shi, Y. M.; Zhang, H.; et al. Growth of Large-Area and Highly Crystalline $\mathrm{MoS}_{2}$ Thin Layers on Insulating Substrates. Nano Lett. 2012, 12, 1538-1544.

23. Petrone, N.; Dean, C. R.; Meric, I.; van der Zande, A. M.; Huang, P. Y.; Wang, L.; Muller, D.; Shepard, K. L.; Hone, J. Chemical Vapor Deposition-Derived Graphene with 
Electrical Performance of Exfoliated Graphene. Nano Lett. 2012, 12, 2751-2756.

24. Benameur, M. M.; Radisavljevic, B.; Heron, J. S.; Sahoo, S.; Berger, H.; Kis, A. Visibility of Dichalcogenide Nanolayers. Nanotechnology 2011, 22, 125706.

25. Brivio, J.; Alexander, D. T. L.; Kis, A. Ripples and Layers in Ultrathin $\mathrm{MoS}_{2}$ Membranes. Nano Lett. 2011, 11, 51485153.

26. Kowalczyk, S. W.; Grosberg, A. Y.; Rabin, Y.; Dekker, C. Modeling the Conductance and DNA Blockade of SolidState Nanopores. Nanotechnology 2011, 22, 315101.

27. Raillon, C.; Granjon, P.; Graf, M.; Steinbock, L. J.; Radenovic, A. Fast and Automatic Processing of Multi-Level Events in Nanopore Translocation Experiments. Nanoscale 2012, 4, 4916-4924.

28. Meller, A.; Nivon, L.; Branton, D. Voltage-Driven DNA Translocations through a Nanopore. Phys. Rev. Lett. 2001, 86, 3435-3438.

29. Fologea, D.; Gershow, M.; Ledden, B.; McNabb, D. S.; Golovchenko, J. A.; Li, J. L. Detecting Single Stranded DNA with a Solid State Nanopore. Nano Lett. 2005, 5, 1905-1909.

30. Fologea, D.; Brandin, E.; Uplinger, J.; Branton, D.; Li, J. DNA Conformation and Base Number Simultaneously Determined in a Nanopore. Electrophoresis 2007, 28, 31863192.

31. Li, J. L.; Gershow, M.; Stein, D.; Brandin, E.; Golovchenko, J. A. DNA Molecules and Configurations in a Solid-State Nanopore Microscope. Nat. Mater. 2003, 2, 611-615.

32. Tabard-Cossa, V.; Trivedi, D.; Wiggin, M.; Jetha, N. N.; Marziali, A. Noise Analysis and Reduction in Solid-State Nanopores. Nanotechnology 2007, 18, 305505.

33. Raillon, C.; Cousin, P.; Traversi, F.; Garcia-Cordero, E.; Hernandez, N.; Radenovic, A. Nanopore Detection of Single Molecule RNAP-DNA Transcription Complex. Nano Lett. 2012, 12, 1157-1164.

34. Storm, A. J.; Chen, J. H.; Ling, X. S.; Zandbergen, H. W.; Dekker, C. Fabrication of Solid-State Nanopores with Single-Nanometre Precision. Nat. Mater. 2003, 2, 537-540.

35. Kim, M. J.; Wanunu, M.; Bell, D. C.; Meller, A. Rapid Fabrication of Uniformly Sized Nanopores and Nanopore Arrays for Parallel DNA Analysis. Adv. Mater. 2006, 18, 3149-3153.

36. Wu, M. Y.; Smeets, R. M. M.; Zandbergen, M.; Ziese, U.; Krapf, D.; Batson, P. E.; Dekker, N. H.; Dekker, C.; Zandbergen, H. W. Control of Shape and Material Composition of Solid-State Nanopores. Nano Lett. 2009, 9, 479-484.

37. Radenovic, A.; Trepagnier, E.; Csencsits, R.; Downing, K. H.; Liphardt, J. Fabrication of $10 \mathrm{~nm}$ Diameter Hydrocarbon Nanopores. Appl. Phys. Lett. 2008, 93, 183101. 